\title{
Multiple Renal Arteries: Variations Demonstrated by Multidetector Computed Tomography Angiography
}

\author{
Mustafa Koplay ${ }^{a}$ Omer Onbas ${ }^{c}$ Fatih Alper ${ }^{c}$ Erim Gulcan ${ }^{b}$ Mecit Kantarcic \\ aDepartment of Radiology, Faculty of Medicine, Selçuk University, Konya, and Departments of ${ }^{\mathrm{b}}$ Internal Medicine, \\ Division of Nephrology, and ${ }^{\mathrm{C}}$ Radiology, Faculty of Medicine, Atatürk University, Erzurum, Turkey
}

\section{Key Words}

Multiple renal arteries $\cdot$ Multidetector computed

tomography angiography

\begin{abstract}
Objective: To present a case of a rare variation of the renal artery and multiple (7) renal arteries by multidetector computed tomography (MDCT) angiography. Clinical Presentation and Intervention: A 36-year-old male patient was admitted to our hospital as a potential living donor for renal transplantation. An MDCT angiography was performed using a 16-detector row CT scanner to obtain a detailed image of vascular structures and associated pathologies. The MDCT clearly revealed the presence of 3 right and 2 left renal arteries arising from the abdominal aorta. Additionally, the accessory renal artery arose from the inferior mesenteric artery on the left side and from the common iliac artery on the right side. Conclusion: This case highlights the importance of awareness of renal artery variations if surgical procedures are indicated in this region.

Copyright $\odot 2010$ S. Karger AG, Basel
\end{abstract}

\section{Introduction}

Renal arteries typically arise at the level of the upper margin of the second lumbar vertebral body, $1 \mathrm{~cm}$ below the origin of the superior mesenteric artery [1]. However, the majority of published data on renal artery anatomy are based on postmortem observations or arteriograms [2]. It is important to be aware of the location and anatomy of the renal vascular pedicle during surgical procedures and invasive interventions. To obtain an accurate diagnosis during radiologic procedures and to perform complication-free surgery, knowledge of the individual's anatomy and potential congenital anomalies are paramount [3].

Color Doppler ultrasonography (US), computed tomography angiography (CTA), magnetic resonance angiography (MRA) and renal arteriography have been used to evaluate and image renal arteries in vivo. Multidetector scanners have advanced the imaging clarity in computed tomography, making it possible to visualize vascular structures in detail. Multidetector computed tomography (MDCT) is a reliable and noninvasive tool for diagnosing renal artery variations and pathologies [4]. We present a case of a rare arterial variation in whom the renal artery arose from the inferior mesenteric artery (IMA) and common iliac artery (CIA).

\section{KARGER}

Fax +41613061234

E-Mail karger@karger.ch

www.karger.com
(C) 2010 S. Karger AG, Basel

1011-7571/10/0195-0412\$26.00/0

Accessible online at:

www.karger.com/mpp
Erim Gülcan

Department of Internal Medicine, Division of Nephrology, Atatürk University Medical Faculty, The Central Campus, Erzurum (Turkey)

Tel. +90 2742652031 ext. 2731, Fax +90 2742652277

E-Mail drerimgulcan@gmail.com 


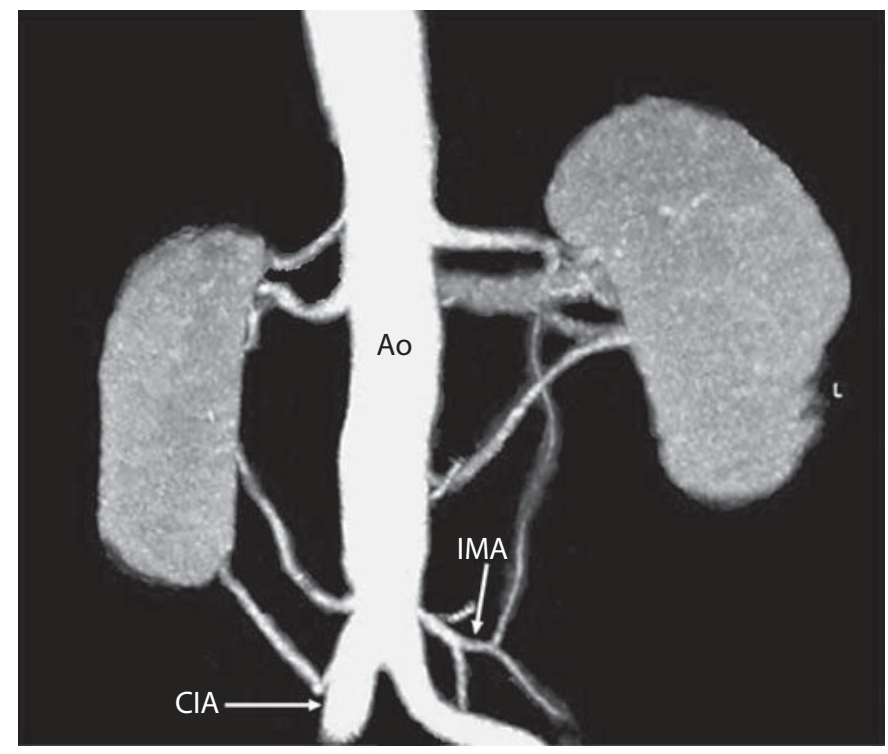

Fig. 1. MIP image of the kidney obtained with MDCT angiography showed 2 normal kidneys with the presence of 3 right renal arteries and 2 left renal arteries arising from the abdominal aorta and the accessory renal artery arising from the IMA (left-sided) and CIA (right-sided). Ao = Aorta.

\section{Case Report}

A healthy 36-year-old male with normal renal function was evaluated with MDCT angiography as a potential living donor for renal transplantation. He had no history of renal artery disease and physical examination and abdominal US were normal. Laboratory tests included evaluations of renal function (e.g. urea, creatinine) and these findings were all normal. Filtration function was measured with intravenous pyelography and was found to be normal in both kidneys.

Detailed images of the vascular structures and pathologies were obtained using MDCT with a 16-detector row CT scanner (Aquillon, Toshiba Medical Systems, Tokyo, Japan). Scans were obtained with $16 \times 0.5 \mathrm{~mm}$ collimation, $1.0 \mathrm{~mm}$ slice thickness, and $1.0 \mathrm{~mm}$ reconstruction interval. Immediately prior to the scan, $100 \mathrm{ml}$ of iodinated contrast material was injected through the antecubital vein at a rate of $4 \mathrm{ml} / \mathrm{s}$. Arterial phase volumetric data sets were acquired for $20 \mathrm{~s}$ from the start of the intravenous injection of the contrast material. All image data were reconstructed with the body soft tissue algorithm.

Reconstructed images were then transferred to a processing workstation for further analysis using specialized software (Vitrea 2, Vital Images, Inc., Minneapolis, Minn., USA). In addition to traditional axial images, multiplanar reconstructions, maximum intensity projection (MIP) and three-dimensional volume rendering ( $3 \mathrm{D} \mathrm{VR}$ ) images were used to assess the structure of the renal arteries. The 3D VR and MIP images of the kidneys obtained using MDCT angiography revealed normal kidneys with the presence of 3 right renal arteries and 2 left renal arteries which arose from the abdominal aorta. Accessory renal arteries arose from the IMA on the left side and the CIA on the right side (fig. 1), making it a total of 7 renal arteries. The sizes of the right and left kidneys were $115 \times 45 \mathrm{~mm}$ and $125 \times 55 \mathrm{~mm}$, respectively. Diameters of the arteries on the right side were $3,8,3$ and $4 \mathrm{~mm}$, superiorly to inferiorly. Diameters of the arteries on the left side were 9, 4 and $2 \mathrm{~mm}$, superiorly to inferiorly. Based on these findings, transplantation could not be performed due to the numerous thin vessels.

\section{Discussion}

Accessory renal arteries are not a rare renal vascular variation and can be found in up to one third of patients. These accessory arteries represent the vestiges of the embryologic blood supply to the kidneys [5, 6]. Multiple renal arteries may occur by the persistence of some arteries from the urogenital rete arteriosum. These can either be an accessory hilar renal artery, with a diameter similar to the renal artery, or accessory vessels to the upper and/or lower renal poles, which usually have a diameter that is smaller than the main renal artery [5]. In a cadaver study, Pollak et al. [7] reported that $23 \%$ had double renal arteries, $4 \%$ had triple arteries, and $1 \%$ had quadruple arteries. Multiple renal arteries occur on the left side in 26-32\% and on the right side in $23-29 \%$ of people $[1,7]$. Bilateral multiple renal arteries occur in $10 \%$ of the population [6]. Accessory renal arteries often arise from the abdominal aorta [8]. Rarely, they arise from the celiac trunk, superior mesenteric arteries, inferior mesenteric arteries, the area near the aortic bifurcation, the common iliac arteries or the median sacral artery $[1,6,8]$. The majority $(70-$ $75 \%$ ) of people may be expected to have 1 renal artery on each side, with the remainder being expected to have 2 or more renal arteries on each side $[1,7]$. In our case, accessory renal arteries arose from the abdominal aorta $(3$ on the right side and 2 on the left side) and CIA ( 1 on the right side) and IMA (1 on the left side).

Knowledge of the presence and location of renal artery variations are necessary when performing renal and adrenal arteriography. Balloon angioplasty and stent implantation are common for the treatment of renal artery pathology, but these procedures may be negatively impacted by the presence of arterial variations [2]. The diagnostic methods for the detection of renal artery variants are renal arteriography, color Doppler US, CTA and MRA. Renal arteriography is an invasive and expensive method. Therefore, noninvasive techniques such as color Doppler US, CTA and MRA have become more widely used.

Color Doppler US is difficult and time-consuming [9]. It is an operator-dependent test that may not be accurate 
when evaluating obese patients. MRA is of limited value when the patient is uncooperative and it is not a preferred method due to high cost and prolonged scan time. Additionally, MRA does not provide optimal imaging of the abdominal region because it is significantly affected by movement artifact associated with breathing. The disadvantages associated with other forms of imaging have made MDCT the gold standard for most clinical applications. MDCT is a reliable, easily applicable and noninvasive tool for visualization of abdominal organs and vascular structures [10]. Multiplanar and 3D VR reconstructions can improve the anatomic details for clinicians and, in selected instances, provide additional information on the nature and extent of disease [11]. In our case, renal artery disease was not found, but multiple renal artery variations were detected.

\section{Conclusion}

This case highlights the importance of the awareness of the presence of renal artery variants if surgical procedures are indicated in this region. Moreover, MDCT can reveal pathologies of the renal arteries and/or of the kidneys.

\section{References}

$>1$ Kawamoto S, Montgomery RA, Lawler LP, Horton KM, Fishman EK: Multidetector row CT evaluation of living renal donors prior to laparoscopic nephrectomy. Radiographics 2004;24:453-466.

$>2$ Beregi JP, Mauroy B, Willoteaux S, MounierVehier C, Rémy-Jardin M, Francke J: Anatomic variation in the origin of the main renal arteries: spiral CTA evaluation. Eur Radiol 1999;9:1330-1334.

-3 Turgut HB, Bircan MK, Hatipoglu ES, Dogruyol S: Congenital anomalies of left renal vein and its clinical importance: a case report and review of literature. Clin Anat 1996;9:133-135.
4 Fraioli F, Catalano C, Bertoletti L, Danti M, Fanelli F, Napoli A, et al: Multidetector-row CT angiography of renal artery stenosis in 50 consecutive patients: prospective interobserver comparison with DSA. Radiol Med 2006;111:459-468.

5 Rossi UG, Romano M, Ferro C: Seven renal arteries. Clin Anat 2006;19:632-633.

6 Urban BA, Ratner LE, Fishman EK: Threedimensional volume-rendered CT angiography of the renal arteries and veins: normal anatomy, variants, and clinical application. Radiographics 2001;21:373-386.

-7 Pollak R, Prusak BF, Mozes MF: Anatomic abnormalities of cadaver kidneys procured for purposes of transplantation. Am Surg 1986;52:233-235.

$\checkmark 8$ Pozniak MA, Balison DJ, Lee FT, Tambeaux RH, Uehling DT, Moon TD: CT angiography of potential renal transplant donors. Radiographics 1998;18:565-587.
9 Kraus GJ, Goerzer HG: MR-angiographic diagnosis of an aberrant retroaortic left renal vein and review of the literature. Clin Imaging 2003;27:132-134.

10 Hyare H, Desigan S, Nicholl H, Guiney MJ, Brookes JA, Lees WR: Multisection CT angiography compared with digital subtraction angiography in diagnosing major arterial hemorrhage in inflammatory pancreatic disease. Eur J Radiol 2006;59:295-300.

11 Kawamoto S, Lawler LP, Fishman EK: Evaluation of the renal venous system on late arterial and venous phase images with MDCT angiography in potential living laparoscopic renal donors. AJR Am J Roentgenol 2005; 184:539-545. 